\title{
The role of magnification correction in macular vessel density assessment: a contralateral eye study in anisometropia patients
}

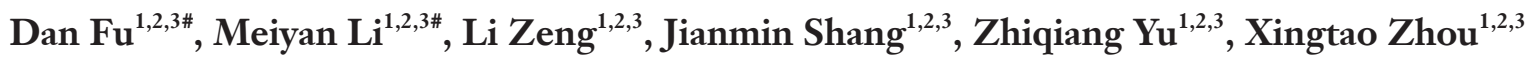 \\ ${ }^{1}$ Eye Institute and Department of Ophthalmology, Eye \& ENT Hospital, Fudan University, Shanghai, China; ${ }^{2}$ NHC Key Laboratory of \\ Myopia (Fudan University), Key Laboratory of Myopia, Chinese Academy of Medical Science, Shanghai, China; ${ }^{3}$ Shanghai Research Center of \\ Ophthalmology and Optometry, Shanghai, China \\ Contributions: (I) Conception and design: X Zhou, Z Yu; (II) Administrative support: X Zhou; (III) Provision of study materials or patients: D Fu, X \\ Zhou; (IV) Collection and assembly of data: M Li, J Shang; (V) Data analysis and interpretation: L Zeng; (VI) Manuscript writing: All authors; (VII) \\ Final approval of manuscript: All authors. \\ \#These authors contributed equally to this work. \\ Correspondence to: Xingtao Zhou. Department of Ophthalmology and Vision Science, Eye and ENT Hospital of Fudan University, No. 83 Fenyang \\ Road, Shanghai 200031, China. Email: doctzhouxingtao@163.com.
}

\begin{abstract}
Background: Investigating the impact of magnification correction in macular vessel density using optical coherence tomography angiography (OCTA) in patients with anisometropia.

Methods: Cross-sectional study. Totally 47 patients (11 male, 36 female) aged $>18$ years with high myopia were analyzed. All patients underwent evaluation of visual acuity, subjective refraction, and axial length. Anisometropia ( $n=37$ ) was defined as a refraction difference between paired eyes $\geq 0.75 \mathrm{D}$. The control group $(\mathrm{n}=10)$ consisted patients with a refraction difference $\leq 0.5$ D. Superficial vessel density was performed using $3 \mathrm{~mm} \times 3 \mathrm{~mm}$ Cirrus-HD OCTA protocol. The vessel length density (VLD) and foveal avascular zone area (FAZA) were analyzed before and after magnification correction using Bennett's formula.
\end{abstract}

Results: The mean spherical equivalent (SE) was $-10.54 \pm 3.47 \mathrm{D}$ in the more myopic eye and $-8.05 \pm 3.47 \mathrm{D}$ in the contralateral eye $(\mathrm{P}<0.001)$. Before magnification correction, the mean perfusion density (PD) and VLD were both significantly lower in the more myopic eyes. After magnification correction, the VLD and PD did not differ between paired eyes. No statistical difference was found in terms of the FAZA between paired eyes regardless of magnification correction. The magnification-induced differences in both VLD and PD were positively correlated with the difference in $\mathrm{SE}$ (both $\mathrm{r}=0.86, \mathrm{P}<0.001$ ).

Conclusions: In OCTA analysis, magnification correction should be performed to reduce refraction errorinduced image error, which deserves attention in the clinical application.

Keywords: Anisometropia; magnification correction; high myopia; vessel density; optical coherence tomography angiography (OCTA)

Submitted Aug 05, 2020. Accepted for publication Nov 27, 2020.

doi: $10.21037 / \mathrm{atm}-20-5698$

View this article at: http://dx.doi.org/10.21037/atm-20-5698

\section{Introduction}

Myopia, which has recently undergone an unprecedented rise, becomes a global health problem (1). It is estimated that by 2050 approximately $50 \%$ of the world's population may be myopic, and that $10 \%$ of the population may be highly myopic (2). Though there are several refractive surgeries to correct myopia (3). Controlling myopia progression is always the most paramount topic. Strategies such as spectacles, contact lenses, orthokeratology, and pharmaceutical treatment are applied worldwide $(4,5)$. Because with the progression of myopia, risks of various ocular pathologies increase, including but not limited to cataracts, glaucoma, retinal detachment, and macular 
degeneration (6).

Optical coherence tomography angiography (OCTA) enables $3 \mathrm{D}$ visualization of the microvasculature by performing repeated $\mathrm{B}$-scans to detect motion contrast. Multiple repeated B-scans are compared pixel by pixel to detect signal changes which occur due to flowing erythrocyte (7). OCTA has the advantage of depth resolution, high contrast, well-defined image and noninvasive fashion. Up to now, OCTA is applied in several ocular pathology diagnosis, such as uveitis, cornea vascularization and retinal disease (8-10).

It has been reported that the average retinal blood flow and vessel diameter were significantly decreased in highly myopic eyes compared to normal eyes $(11,12)$. However, identical vessel density in anisometropia induced amblyopic eyes also has been observed (13). These inconsistent results inspired us to search for the root cause. Besides the effect of amblyopia, the error originated from magnification correction might partly explain these varied results. Because the error caused by magnification correction could result in variation of $-20 \%$ to $+10 \%$ in vessel density values (14). Furthermore, most studies investigated differences between groups, but deviations among individuals were relatively high.

Notably, anisometropia is a natural model for withinpatient comparisons, which minimizes the effects of individual differences. The principle of treating anisometropia is to restore balanced sensory ocular dominance between paired eyes (15). Effective strategies include refractive surgery and rigid gas permeable $(16,17)$. Refractive anisometropia develops primarily through the influence of changes in the posterior segment of the eye, as indicated by previous histology and population-based studies $(18,19)$. However, the differences in bilateral fundus pathology, which is particularly crucial in patients with high myopia, are not yet fully characterized. For anisometropic eyes, the impact of varying ocular size on fundus pathology is unknown.

Thus, using the model of anisometropia, we aimed to investigate the role of magnification correction in vessel density analysis using OCTA, which may aid understanding anisometropia's pathology.

We present the study in accordance with the MDAR reporting checklist (available at http://dx.doi.org/10.21037/ atm-20-5698).

\section{Methods}

This study was approved by the Ethics Committee of the Eye,
Ear, Nose, and Throat (EENT) Hospital of Fudan University (No. 2016036) and was performed in accordance with the principles of the Declaration of Helsinki (as revised in 2013). Written informed consent was acquired from each patient.

Between August 2017 and December 2017, a series of anisometropic patients from the Refractive Center of the EENT Hospital of Fudan University were recruited. All patients underwent thorough examinations including evaluation of visual acuity [logarithm of the minimal angle of resolution ( $\log M A R)]$, subjective refraction, and axial length (Intraocular Lens Master, Carl Zeiss Meditec, Jena, Germany), indirect dilated fundus ophthalmoscopy, and fundus photography. Patients with anisometropia were screened using the following inclusion criteria: difference in spherical equivalent (SE) ( $\triangle \mathrm{SE}, \mathrm{SE}$ in the more myopic eye minus that of the less myopic eye) between two eyes of at least $-0.75 \mathrm{D}$, at least one eye with high myopia (SE $\leq-6.0 \mathrm{D})$, cylinder refraction difference $\leq 1.5 \mathrm{D}$, and corrected distance visual acuity (CDVA) $\geq 20 / 25$. The exclusion criteria were as follows: hyperopia, degenerative macular disease or retinal deterioration, poor OCT image quality resulting from unstable fixation, history of ocular surgery, severe cataracts, glaucoma, or retinal detachment, or systemic disease such as diabetes mellitus.

Patients with $\triangle \mathrm{SE}$ of $-0.5 \mathrm{D}$ or less were enrolled as the control group.

\section{OCT scanning}

ZEISS AngioPlexTM OCT (AngioPlex software, version 10.0; USA) was used to acquire high-resolution images of the retinal microvasculature. The procedures and assessments were in accordance with those of a previous study (20). Vessel length density (VLD, defined as the total length of perfused vasculature per unit area in a region of measurement) and perfusion density (PD, defined as the total area of perfused vasculature per unit in a region of measurement) were assessed via the prototype algorithm provided by Zeiss Meditec. One standard $3 \mathrm{~mm} \times 3 \mathrm{~mm}$ scanning pattern was used for all patients. In accordance with the Early Treatment of Diabetic Retinopathy Study, vessel density was quantified in a set of five regions (Figure 1), thereby automatically producing a mean value of the entire scan area. Presently, the software only reported vessel density values in the superficial capillary plexus, which extends from the outer boundary of the inner limiting membrane to the outer boundary of the inner plexiform layer. The foveal avascular zone area (FAZA) is 


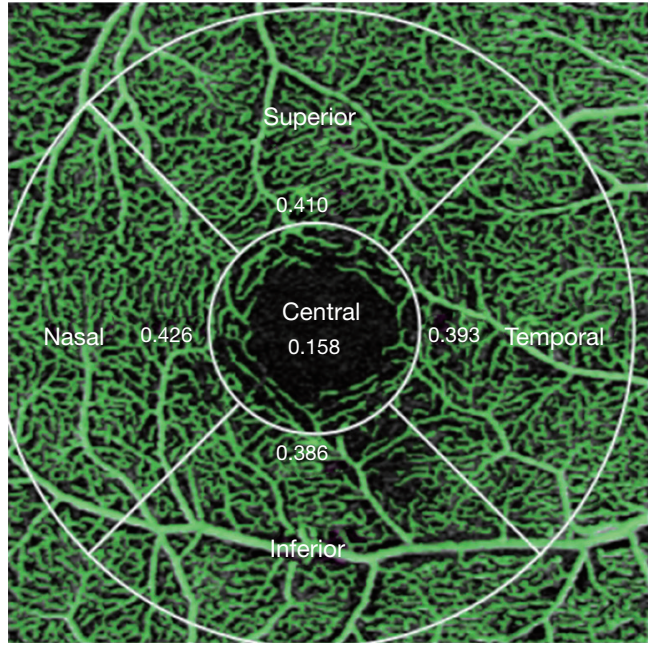

Figure 1 An angiography scan mode in ETDRS analysis in CirrusHD OCTA. OCTA, optical coherence tomography angiography.

automatically outlined and calculated.

Each scan was repeated three times, and patients were instructed to close their eyes for $30 \mathrm{~s}$ at the interval. The scan with best signal strength was used for analysis, and the prior scan was used if two or three images had the same signal strength. All procedures were performed under the same illumination by one trained examiner, and only images with signal strength of $\geq 7$ points (full score is 10 points) were included in the analysis.

To account for the differences in axial length in the anisometric cohort, a transverse OCT scaling factor based on axial length was calculated for each eye individually. Images were corrected for magnification using Bennett's formula (21). The use of axial length was demonstrated to be the simplest and most accurate method of magnification correction (22). The relationship between a measured $\mathrm{SD}-\mathrm{OCT}$ image (the measurement circle in this case) and its actual size can be expressed as $\mathrm{t}=\mathrm{p} \times \mathrm{q} \times \mathrm{s}$, where $t$ is the actual size of an object on the retina, $s$ is the SDOCT measurement, $\mathrm{p}$ is the magnification factor related to the OCT's camera [3.382 in Cirrus HD (23)], and q is the magnification factor related to the eye $[q=0.01306 \times$ (axial-1.82)].

\section{Statistical analysis}

Data analysis was performed using SPSS 22.0 (SPSS, Inc., Chicago, IL). All data are described as mean \pm standard deviation. A paired $t$-test was used to compare bilateral eyes with a normal distribution, and Pearson's correlation was used to analyze associations between variables. For non-normally distributed data, the Wilcoxon signedrank test and Spearman's rank-order correlation were used. Differences before and after axial correction were determined using a paired $t$-test. $\mathrm{P}$ values of $<0.05$ were considered statistically significant.

\section{Results}

\section{Demographic results}

Among 50 patients, three cases were excluded: one for the presence of dark shadow, and two for unstable fixation. Finally, 47 patients (including ten in the control group) aged 18-47 years were included in the study (11 male, 36 female). Pooling all cases, the mean SE of the more myopic eye was $-10.54 \pm 3.47 \mathrm{D}$, while that of the contralateral eye was $-8.05 \pm 3.47 \mathrm{D}(\mathrm{P}<0.001)$, and the mean $\Delta \mathrm{SE}$ was $-2.49 \pm 2.16 \mathrm{D}$. The average CDVA of the more myopic eye was $-0.03 \pm 0.12 \log M A R$, and that of the contralateral eye was $-0.03 \pm 0.07 \log M A R(P=0.12)$. The baseline information of all patients is provided in Table 1 .

The average $\Delta \mathrm{SE}$ was $-3.15 \pm 2.04 \mathrm{D}$ and $-0.32 \pm 0.29 \mathrm{D}$ in the anisometropic group and control group, respectively $(\mathrm{P}<0.001)$.

\section{OCTA parameters}

The acquired vascular images were clearly visualized in all vascular network maps. The average vessel density, including VLD and PD, is presented in Table 2. Without magnification correction, the mean PD was significantly lower in the more myopic eyes. The VLD was also significantly lower in the more myopic eyes in terms of the inferior, nasal, and mean value.

After magnification correction, the VLD between paired eyes has no difference, while in the superior area, higher $\mathrm{PD}$ was observed in the more myopic eyes $(\mathrm{P}=0.02)$.

The FAZA were $0.26 \pm 0.10$ and $0.24 \pm 0.11 \mathrm{~mm}^{2}$ $(\mathrm{P}=0.87)$ before magnification correction and $0.28 \pm 0.12$ and $0.26 \pm 0.12 \mathrm{~mm}^{2}(\mathrm{P}=0.66)$ after correction in the more myopic and less myopic eyes, respectively.

\section{Subgroup and correlation analysis}

After magnification correction, the significant difference of PD and VLD between paired eyes in the anisometropia 
Table 1 Baseline information of enrolled patients

\begin{tabular}{lccc}
\hline Variables & The more myopic eyes & The less myopic eyes & $P$ \\
\hline Spherical diopters $(\mathrm{D})$ & $-9.84 \pm 3.29$ & $-7.38 \pm 3.42$ & $-1.25 \pm 0.99$ \\
Cylinder $(\mathrm{D})$ & $-1.28 \pm 0.87$ & $26.21 \pm 1.54$ & 0.69 \\
Axial length $(\mathrm{mm})$ & $27.23 \pm 1.78$ & $15.4 \pm 2.5$ & $<0.001$ \\
IOP $(\mathrm{mmHg})$ & $15.6 \pm 2.04$ & 0.54 \\
\hline
\end{tabular}

IOP, intraocular pressure.

Table 2 The average vessel density in each area before and after magnification correction

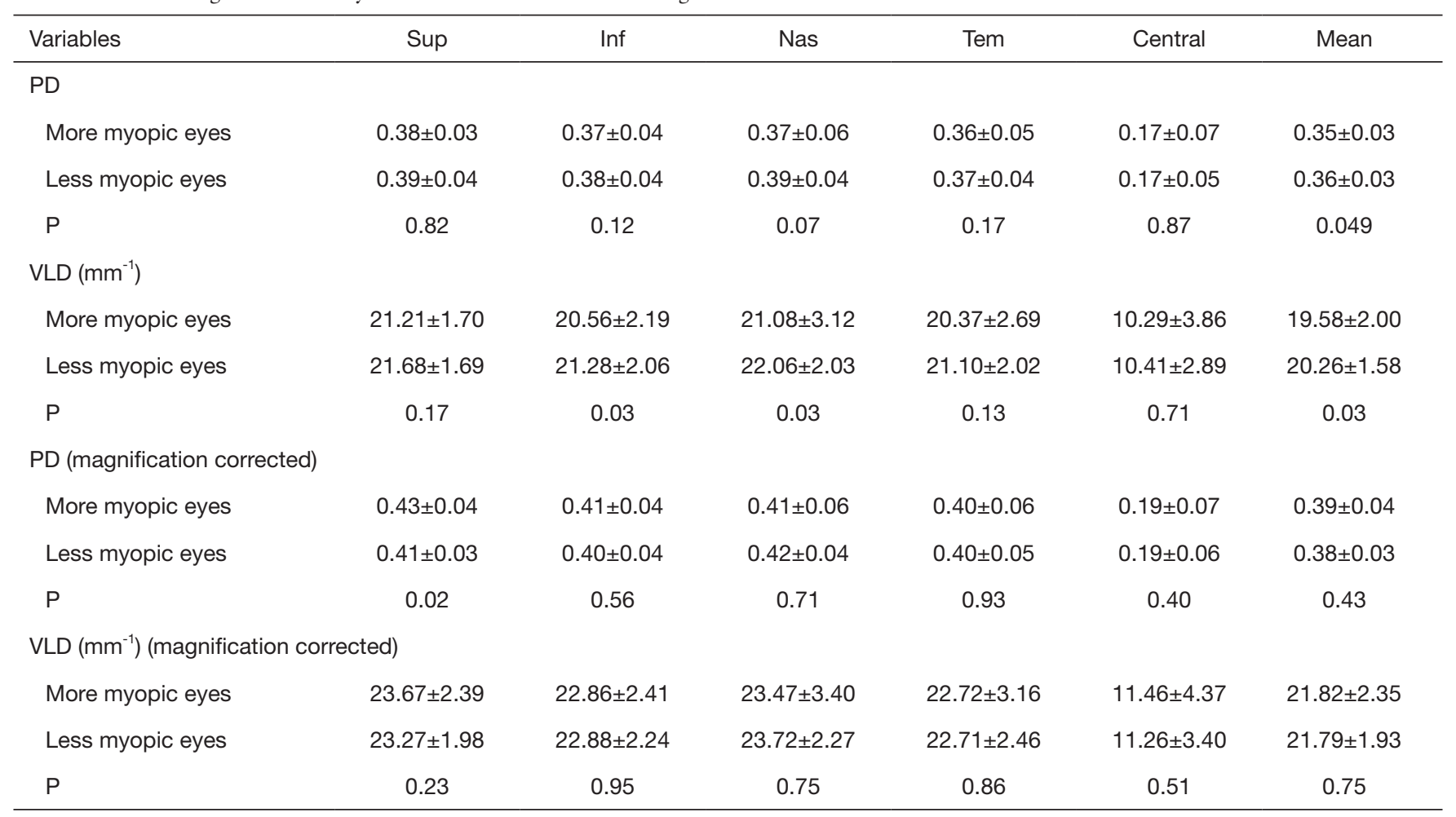

PD, perfusion density; VLD, vessel length density; Sup, superior; Inf, inferior; Nas, nasal; Tem, temporal.

group was eliminated. However, in the control group, the identical values in paired eyes did not change after magnification correction (Figure 2).

We did not identify a correlation between $\triangle \mathrm{SE}$ and vessel density difference $(\triangle \mathrm{VLD} / \mathrm{PD}$, values in the less myopic eyes minus that of the more myopic eyes) before or after correction. However, the magnification-induced error ( $\triangle$ VLDbefore $-\triangle$ VLDafter) increased with the absolute $\Delta \mathrm{SE}(\mathrm{r}=0.86, \mathrm{P}<0.001)$, and similar results were also detected in $\triangle \mathrm{PDb}$ bore $-\triangle \mathrm{PD}$ after with the absolute $\Delta \mathrm{SE}$ $(\mathrm{r}=0.86, \mathrm{P}<0.001)$ (Figure 3).

\section{Discussion}

Based on a previous report of significantly decreased blood flow in highly myopic eyes (24), the present study only enrolled patients with high myopia. Our study demonstrated that magnification correction should be performed in retinal vessel density analysis.

The present study indicated that magnification correction with Bennett's formula eliminates the difference in vessel density between paired eyes. The trend becomes more apparent with increasing anisometropia. According to Bennett's formula, we can calculate that an axial length 


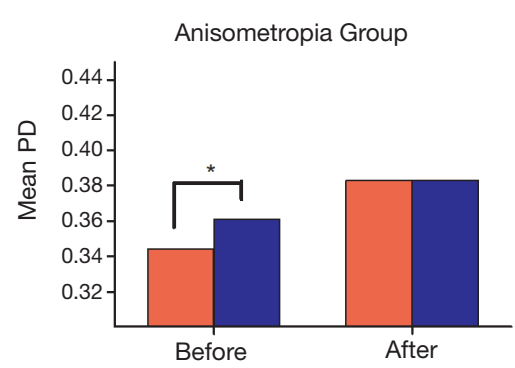

Anisometropia Group

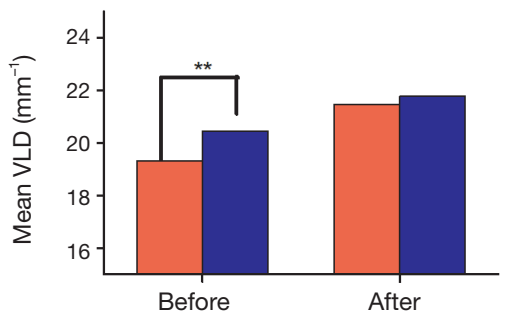

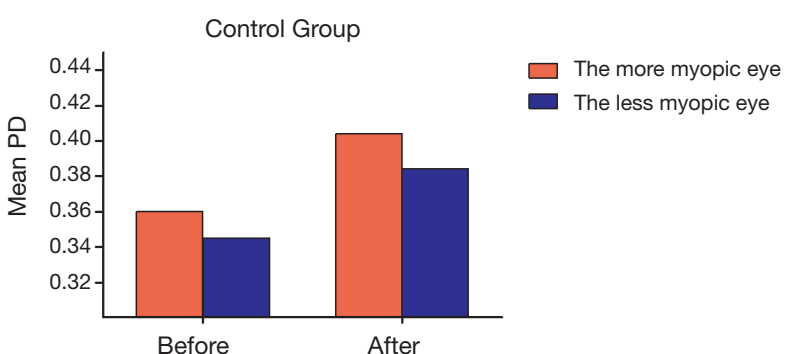

Control Group

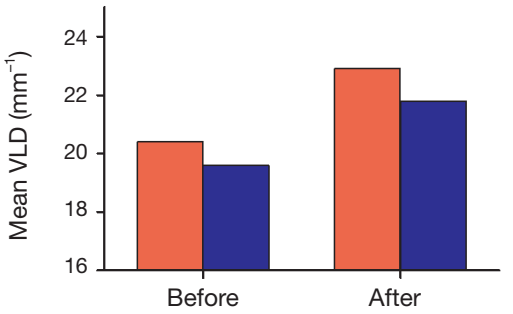

Figure 2 Paired comparisons of vessel density before and after magnification correction. PD, perfusion density; VLD, vessel length density. *, $\mathrm{P}<0.05 ;{ }^{* *}, \mathrm{P}<0.01$.
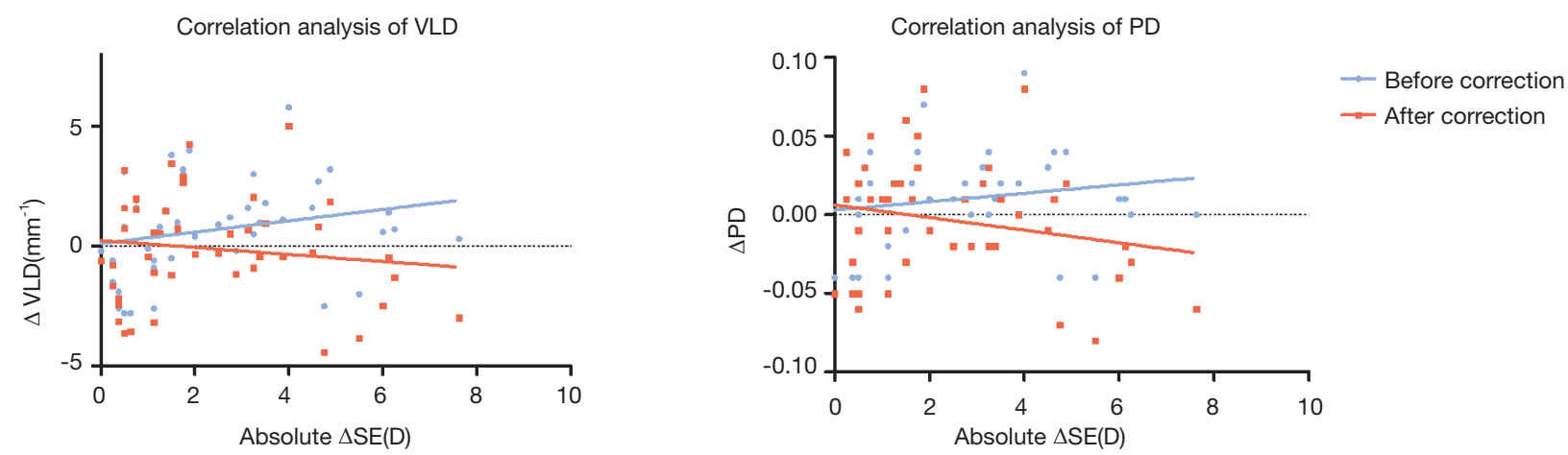

Figure 3 Correlation analysis between $\Delta$ vessel density and absolute $\Delta$ spherical equivalent. PD, perfusion density; VLD, vessel length density.

of $>24.46 \mathrm{~mm}$ results in increased vessel density following magnification correction. Since the enrolled eyes were highly myopic with axial lengths of $>26 \mathrm{~mm}$, magnification correction resulted in increased vessel density and the difference between paired eyes decreased. These results were consistent with those of the study by Sampson et al. (14), despite the use of different OCT techniques.

Without magnification correction, vessel density was generally lower in eyes with higher myopia, which is in accordance with previous study (25). With magnification correction, there are some conflicting results. Yang et al. (26) found that the large and micro vessel density in the retina declined in highly myopic eyes, while Cheung et al. (27) found greater vessel density in eyes with longer axial lengths. We found no difference in macular vessel density with varying axial length. In addition to the different scanners and calculation algorithms used in different studies, the varying inclusion criteria might also have contributed to this discrepancy. Cheung's study enrolled patients with continuous refractive error, and in Yang's study, the mean difference in SE between the high myopia and control groups was approximately $8.0 \mathrm{D}$, while 
in our study, the mean difference in SE was $2.5 \mathrm{D}$ between paired eyes. Thus, we speculate there may exist a refractive error threshold value that can be used to distinguish vessel density; however, this requires confirmation in further study.

In terms of anisometropia, the current results revealed no difference in vessel density in paired eyes. This indicates that the retina is not the only target of ocular growth modulation. This is consistent with the findings of Huang et al. (28), who found that, in addition to producing central refractive errors, abnormal visual experience can alter the shape of the posterior globe and cause peripheral refractive errors in infant primates. The vitreous chamber, for example, can be modulated to correct for refractive or axial anisometropia.

The balanced vessel density distribution is in accordance with the identical visual acuity observed in the current study. Lonngi et al. (29) observed significantly decreased vessel density in patients with amblyopia. Demirayak et al. (30) also found lower vessel density in amblyopic eyes than fellow eyes. Amblyopia means different corrected visual acuity in paired eyes. That is the key point that distinguishes our study from the two references above, resulting to no difference in vessel density between the eyes. This result inspired the hypothesis that visual acuity can be an indicator of retinal vessel circulation change. Similar results were derived from Liu's study (31) that vessel density was a prominent factor affecting visual acuity. The underlying mechanism may be that nerve fiber and ganglion cell layer receive blood from the superficial retinal vascular plexus and the degree of perfusion may impact visual performance (32). This indicated that both refraction error and visual acuity should be considered when assessing retinal condition (33).

In addition to vessel density, other OCT findings are affected by magnification correction. Retinal nerve fiber thickness (RNFL) was considered to be negatively correlated with axial length, while the correlation was eliminated after magnification correction in the study by Kausar (34). Using the same scanner (Cirrus HD-OCT) as that used in the present study, Savini et al. (35) found that correcting for axial length resolved the relationship between axial length and the RNFL and optic nerve head. Moghimi et al. (36) concluded that ocular magnification is necessary in optical disc and neuroretinal rim area measurement. The present study findings are in agreement with previous studies that axial length affects the measurement values of retinal parameters and should be considered in retinal analysis.

We did not detect differences in the FAZA between paired eyes before and after magnification correction. This is consistent with Fujiwara's (37) conclusion that the FAZA is associated with vessel density rather than axial length. One possible reason is that the enrolled eyes were high myopic without pathological changes. We speculate that magnification would have a greater impact in diseased eyes, because pathologic capillary dropout can alter the boundary of the FAZ as well as its size.

The current study has several limitations. One is the relatively small sample size, from which several cases were excluded due to unstable fixation or low signal strength (26). Further studies comparing the vessels of the deep or middle layers of the retina are recommended. The $\Delta \mathrm{SE}$ values used to categorize patients into the anisometropia and control groups were arbitrary. Different threshold values may yield different results, but we speculate that the overall trend will remain unchanged. Studies with a larger sample size and more detailed analyses are warranted.

\section{Conclusions}

In summary, for macular vascular image analysis, differences in image magnification due to different refractive errors require correction before analysis. No difference was found in paired eyes of anisometropia.

\section{Acknowledgments}

Funding: This work was supported by National Natural Science Foundation of China (Grant No. 81770955), Project of Shanghai Science and Technology (Grant No. 17411950200), Joint research project of new frontier technology in municipal hospitals (SHDC12018103).

\section{Footnote}

Reporting Checklist: The authors have completed the MDAR reporting checklist. Available at http://dx.doi.org/10.21037/ atm-20-5698

Data Sharing Statement: Available at http://dx.doi. org/10.21037/atm-20-5698

Peer Review File: Available at http://dx.doi.org/10.21037/ atm-20-5698 
Conflicts of Interest: All authors have completed the ICMJE uniform disclosure form (available at http://dx.doi. org/10.21037/atm-20-5698). The authors have no conflicts of interest to declare.

Etbical Statement: The authors are accountable for all aspects of the work in ensuring that questions related to the accuracy or integrity of any part of the work are appropriately investigated and resolved. This study was approved by the Ethics Committee of the Eye, Ear, Nose, and Throat (EENT) Hospital of Fudan University (No. 2016036) and was performed in accordance with the principles of the Declaration of Helsinki (as revised in 2013). Written informed consent was acquired from each patient.

Open Access Statement: This is an Open Access article distributed in accordance with the Creative Commons Attribution-NonCommercial-NoDerivs 4.0 International License (CC BY-NC-ND 4.0), which permits the noncommercial replication and distribution of the article with the strict proviso that no changes or edits are made and the original work is properly cited (including links to both the formal publication through the relevant DOI and the license). See: https://creativecommons.org/licenses/by-nc-nd/4.0/.

\section{References}

1. Dolgin E. The myopia boom. Nature 2015;519:276-8.

2. Holden BA, Fricke TR, Wilson DA, et al. Global Prevalence of Myopia and High Myopia and Temporal Trends from 2000 through 2050. Ophthalmology 2016;123:1036-42.

3. Kim TI, Alió Del Barrio JL, Wilkins M, et al. Refractive surgery. Lancet 2019;393:2085-98.

4. Sankaridurg P, Conrad F, Tran H, et al. Controlling Progression of Myopia: Optical and Pharmaceutical Strategies. Asia Pac J Ophthalmol (Phila) 2018;7:405-14.

5. Cooper J, Tkatchenko AV. A Review of Current Concepts of the Etiology and Treatment of Myopia. Eye Contact Lens 2018;44:231-47.

6. Ikuno Y. Overview of the Complications of High Myopia. Retina 2017;37:2347-51.

7. Spaide RF, Fujimoto JG, Waheed NK, et al. Optical coherence tomography angiography. Prog Retin Eye Res 2018;64:1-55.

8. Arya M, Rashad R, Sorour O, et al. Optical coherence tomography angiography (OCTA) flow speed mapping technology for retinal diseases. Expert Rev Med Devices 2018;15:875-82.

9. Devarajan K, Di Lee W, Ong HS, et al. Vessel density and En-face segmentation of optical coherence tomography angiography to analyse corneal vascularisation in an animal model. Eye Vis (Lond) 2019;6:2 .

10. Dingerkus VLS, Munk MR, Brinkmann MP, et al. Optical coherence tomography angiography (OCTA) as a new diagnostic tool in uveitis. J Ophthalmic Inflamm Infect 2019;9:10.

11. Shimada N, Ohno-Matsui K, Harino S, et al. Reduction of retinal blood flow in high myopia. Graefes Arch Clin Exp Ophthalmol 2004;242:284-8.

12. Zheng Q, Zong Y, Li L, et al. Retinal vessel oxygen saturation and vessel diameter in high myopia. Ophthalmic Physiol Opt 2015;35:562-9.

13. Araki S, Miki A, Goto K, et al. Foveal avascular zone and macular vessel density after correction for magnification error in unilateral amblyopia using optical coherence tomography angiography. BMC Ophthalmol 2019;19:171.

14. Sampson DM, Gong P, An D, et al. Axial Length Variation Impacts on Superficial Retinal Vessel Density and Foveal Avascular Zone Area Measurements Using Optical Coherence Tomography Angiography. Invest Ophthalmol Vis Sci 2017;58:3065-72.

15. Jiang F, Chen Z, Bi H, et al. Association between Ocular Sensory Dominance and Refractive Error Asymmetry. PLoS One 2015;10:e136222.

16. Liu H, Chen Q, Lan F, et al. The Modulation of Laser Refractive Surgery on Sensory Eye Dominance of Anisometropia. J Ophthalmol 2020;2020:3873740.

17. Evans BJ. Orthoptic indications for contact lens wear. Cont Lens Anterior Eye 2006;29:175-81; quiz 211.

18. Shen L, You QS, Xu X, et al. Sclera and choroidal thickness in secondary high axial myopia. Retina 2016;36:1579-85.

19. Jonas JB, Xu L. Histological changes of high axial myopia. Eye (Lond) 2014;28:113-7.

20. Lei J, Durbin MK, Shi Y, et al. Repeatability and Reproducibility of Superficial Macular Retinal Vessel Density Measurements Using Optical Coherence Tomography Angiography En Face Images. JAMA Ophthalmol 2017;135:1092-8.

21. Bennett AG, Rudnicka AR, Edgar DF. Improvements on Littmann's method of determining the size of retinal features by fundus photography. Graefes Arch Clin Exp Ophthalmol 1994;232:361-7.

22. Garway-Heath DF, Rudnicka AR, Lowe T, et al. 
Measurement of optic disc size: equivalence of methods to correct for ocular magnification. Br J Ophthalmol 1998;82:643-9.

23. Yang B, Ye C, Yu M, et al. Optic disc imaging with spectral-domain optical coherence tomography: variability and agreement study with Heidelberg retinal tomograph. Ophthalmology 2012;119:1852-7.

24. Ravalico G, Pastori G, Croce M, et al. Pulsatile ocular blood flow variations with axial length and refractive error. Ophthalmologica 1997;211:271-3.

25. Hassan M, Sadiq MA, Halim MS, et al. Evaluation of macular and peripapillary vessel flow density in eyes with no known pathology using optical coherence tomography angiography. Int J Retina Vitreous 2017;3:27.

26. Yang Y, Wang J, Jiang H, et al. Retinal Microvasculature Alteration in High Myopia. Invest Ophthalmol Vis Sci 2016;57:6020-30.

27. Cheung CY, Li J, Yuan N, et al. Quantitative retinal microvasculature in children using swept-source optical coherence tomography: the Hong Kong Children Eye Study. Br J Ophthalmol 2018. doi: 10.1136/ bjophthalmol-2018-312413.

28. Huang J, Hung LF, Ramamirtham R, et al. Effects of form deprivation on peripheral refractions and ocular shape in infant rhesus monkeys (Macaca mulatta). Invest Ophthalmol Vis Sci 2009;50:4033-44.

29. Lonngi M, Velez FG, Tsui I, et al. Spectral-Domain Optical Coherence Tomographic Angiography in Children With Amblyopia. JAMA Ophthalmol 2017;135:1086-91.

30. Demirayak B, Vural A, Sonbahar O, et al. Analysis of Macular Vessel Density and Foveal Avascular Zone in

Cite this article as: Fu D, Li M, Zeng L, Shang J, Yu Z, Zhou X. The role of magnification correction in macular vessel density assessment: a contralateral eye study in anisometropia patients. Ann Transl Med 2021;9(5):380. doi: 10.21037/atm-20-5698
Adults with Amblyopia. Curr Eye Res 2019;44:1381-5.

31. Liu R, Lu J, Liu Q, et al. Effect of Choroidal Vessel Density on the Ellipsoid Zone and Visual Function in Retinitis Pigmentosa Using Optical Coherence Tomography Angiography. Invest Ophthalmol Vis Sci 2019;60:4328-35.

32. Kur J, Newman EA, Chan-Ling T. Cellular and physiological mechanisms underlying blood flow regulation in the retina and choroid in health and disease. Prog Retin Eye Res 2012;31:377-406.

33. Talu S, Vladutiu C, Lupascu CA. Characterization of human retinal vessel arborisation in normal and amblyopic eyes using multifractal analysis. Int J Ophthalmol 2015;8:996-1002.

34. Kausar A, Akhtar N, Afzal F, et al. Effect of refractive errors/axial length on peripapillary retinal nerve fibre layer thickness (RNFL) measured by Topcon SD-OCT. J Pak Med Assoc 2018;68:1054-9.

35. Savini G, Barboni P, Parisi V, et al. The influence of axial length on retinal nerve fibre layer thickness and opticdisc size measurements by spectral-domain OCT. Br J Ophthalmol 2012;96:57-61.

36. Moghimi S, Hosseini H, Riddle J, et al. Measurement of optic disc size and rim area with spectral-domain OCT and scanning laser ophthalmoscopy. Invest Ophthalmol Vis Sci 2012;53:4519-30.

37. Fujiwara A, Morizane Y, Hosokawa M, et al. Factors affecting foveal avascular zone in healthy eyes: An examination using swept-source optical coherence tomography angiography. PLoS One 2017;12:e0188572. 